\title{
A Shift in Thinking to Reduce Mother-to-Infant Transmission of Hepatitis B
}

\author{
Geoffrey Dusheiko, M.D.
}

The morbidity and mortality that are associated with hepatitis B virus (HBV) infection have been overshadowed by the public health prominence of other infectious diseases, including human immunodeficiency virus (HIV) infection, tuberculosis, and malaria. There is a dawning realization that the disease burden from HBV infection is increasing, despite the existence of an effective vaccine. ${ }^{1}$ The World Health Organization (WHO) estimates that chronic HBV infection results in a quarter million deaths each year in the countries that are eligible for Global Vaccine Alliance (GAVI) support or cofinancing. ${ }^{2}$ A positive status for hepatitis B e antigen ( $\mathrm{HBeAg}$ ) and a maternal HBV DNA level of more than 200,000 IU per milliliter are associated with an increased risk of perinatal HBV infection despite vaccination. ${ }^{3}$ Infection in the neonatal period and childhood remains the leading source of new chronic infections and is a silent precursor to progressive disease. Thus, the prevention of neonatal and childhood infection by effective prophylaxis is crucial.

Schedules of either three or four doses are used for hepatitis B immunization, depending on government policy and national prevalence. Currently, HBV vaccination is most frequently administered as a pentavalent or hexavalent vaccine as part of the Expanded Program on Immunization (EPI), typically in combination with vaccines against diphtheria, tetanus, pertussis, polio, and Haemophilus influenzae type B. The first dose of $\mathrm{HBV}$ vaccine is given at 6 weeks of age to ensure immunogenicity of the combination. Despite recommendations from the WHO, only 92 of 193 countries $(48 \%)$ report administering the vaccine at birth. In sub-Saharan Africa, the vaccine is currently administered at birth in $10 \%$ of neonates, and only 11 of 47 countries in the WHO African region have introduced a regimen of vac- cination at birth. ${ }^{4,5}$ As a result, an estimated 1\% of newborns annually ( $>365,000$ newborns in one model) are infected with HBV at birth in subSaharan Africa; this is twice the incidence of HIV infection among infants in this region. ${ }^{6}$

Paradoxically, GAVI support for combination vaccines within an integrated EPI schedule has unwittingly but undesirably shifted thinking and policy away from HBV vaccination at birth. This gap in vaccine strategy is disadvantageous. Antiviral therapy for pregnant women in the third trimester is effective in reducing vaccine and immune globulin failure in children born to mothers with high levels of viremia, but the HBV DNA level cannot be ascertained in many lowincome countries. ${ }^{7}$

In this issue of the Journal, Jourdain et al. ${ }^{8}$ report the results of a multicenter, double-blind trial of tenofovir disoproxil fumarate (TDF) versus placebo that was conducted in 17 public hospitals in Thailand. TDF and placebo were administered from 28 weeks of gestation to 2 months post partum in 331 women who were positive for HBeAg and the hepatitis B surface antigen (HBsAg). The median HBV DNA level was $8.1 \log _{10}$ IU per milliliter in the TDF group and $7.9 \log _{10}$ IU per milliliter in the placebo group. At enrollment, $90 \%$ of the women in the TDF group and $87 \%$ of those in the placebo group had an HBV DNA level of more than 200,000 IU per milliliter. HIV-positive women were excluded. All the children were given $\mathrm{HBV}$ vaccine and hepatitis B immune globulin at birth. Remarkably, the median time of HBV vaccination was 1.2 hours after birth, and $4 \%$ of infants received vaccine after 4 hours. Four doses of HBV vaccine were thus administered. A total of $2 \%$ of the infants in the placebo group ( 3 of 147 infants) and none of the infants in the TDF group were HBsAg-positive at 6 months 
of age. The most frequent adverse event was an elevation in the alanine aminotransferase level in the mothers (18\% in the TDF group and 15\% in the placebo group).

Although this trial and other studies point to some value for antiviral prophylaxis in preventing mother-to-child transmission, this trial did not prove the superiority of TDF prophylaxis. The low rate of transmission in the placebo group (in contrast to previously reported data from Pan et al. ${ }^{7}$ ) implies a role for the administration of the HBV vaccine within hours after birth to reduce the risk of mother-to-child transmission without additional testing or antiviral therapy in mothers with high levels of viremia. Other factors that were not answered in this trial require understanding: these include the effect of vaccination in infants born to mothers coinfected with HBV and HIV, intrauterine transmission, HBV genotypes, and vaccine escape mutants. (HBV DNA levels in mothers with HBV-HIV coinfection are typically higher than in mothers infected with only HBV; however, women with known HIV infection are given antiretroviral treatment, which also lowers HBV DNA levels, and thus the risk of mother-to-child transmission is, theoretically, reduced.)

Given these data, what is the way forward? Several strategies can reduce the incidence of mother-to-child transmission; whatever is feasible should be applied in various countries. Vaccination, particularly if delayed, may fail to protect infants born to mothers with high serum levels of HBV DNA or HBeAg; the current levels of evidence supporting antiviral therapy with TDF (or possibly lamivudine or telbivudine) to reduce levels of maternal HBV DNA during pregnancy have been accepted by the American Association for the Study of Liver Diseases, the European Association for the Study of the Liver, and the Asian Pacific Association for the Study of the Liver. ${ }^{9-11}$ Preventing the residuum of chronic neonatal infections requires testing for HBsAg and HBV DNA and antiviral treatment or, alternatively, simple measures that bypass additional testing and treatment. It is necessary to analyze regional data to assess the requirements for implementing vaccination at birth, including access to deliveries at institutions such as hospitals or birth centers, rates of skilled birth attendance, delivery in conditions that lack cold-chain refrigeration in rural versus urban areas, and the training of otherwise-unskilled birth attendants to deliver monovalent HBV vaccine at the same time as the vaccines against polio and bacille CalmetteGuérin. ${ }^{12}$ Importantly, the use of monovalent HBV vaccine would also require governmental or nongovernmental (e.g., GAVI) support. HBV vaccination at birth, despite the challenges for poverty-affected countries to deliver vaccination in rural and isolated locales, is feasible. A conjoint mobilization of HIV services to serve persons with chronic HBV infection is required. The trial by Jourdain et al. (which the authors consider statistically "negative") puts down an intriguing marker attesting to the possibility that rapidly phasing in the timely administration of a safe monovalent $\mathrm{HBV}$ vaccine within a few hours after birth could contribute to the interruption of mother-to-child transmission and avert preventable HBV infections in childhood.

Disclosure forms provided by the author are available with the full text of this editorial at NEJM.org.

From the University College London School of Medicine and King's College Hospital, London.

1. Schweitzer A, Horn J, Mikolajczyk RT, Krause G, Ott JJ. Estimations of worldwide prevalence of chronic hepatitis B virus infection: a systematic review of data published between 1965 and 2013. Lancet 2015;386:1546-55.

2. Global hepatitis report, 2017: executive summary. Geneva: World Health Organization, 2017 (http://www.who.int/hepatitis/ publications/global-hepatitis-report2017-executive-summary/en/).

3. Wiseman E, Fraser MA, Holden S, et al. Perinatal transmission of hepatitis B virus: an Australian experience. Med J Aust 2009;190:489-92.

4. Immunization, vaccines and biologicals: data, statistics and graphics. Geneva: World health Organization, 2017 (http://www .who.int/immunization/monitoring_surveillance/data/en).

5. Breakwell L, Tevi-Benissan C, Childs L, Mihigo R, Tohme R. The status of hepatitis B control in the African region. Pan Afr Med J 2017;27:Suppl 3:17.

6. Keane E, Funk AL, Shimakawa Y. Systematic review with meta-analysis: the risk of mother-to-child transmission of hepatitis B virus infection in sub-Saharan Africa. Aliment Pharmacol Ther 2016;44:1005-17.

7. Pan CQ, Duan Z, Dai E, et al. Tenofovir to prevent hepatitis B transmission in mothers with high viral load. $N$ Engl J Med 2016;374:2324-34.

8. Jourdain G, Ngo-Giang-Huong N, Harrison L, et al. Tenofovir versus placebo to prevent perinatal transmission of hepatitis B. N Engl J Med 2018;378:911-23.

9. Terrault NA, Bzowej NH, Chang KM, Hwang JP, Jonas MM, Murad MH. AASLD guidelines for treatment of chronic hepatitis B. Hepatology 2016;63:261-83.

10. European Association for the Study of the Liver. EASL 2017 clinical practice guidelines on the management of hepatitis B virus infection. J Hepatol 2017;67:370-98.

11. Sarin SK, Kumar M, Lau GK, et al. Asian-Pacific clinical practice guidelines on the management of hepatitis B: a 2015 update. Hepatol Int 2016;10:1-98.

12. Spearman CW, Afihene M, Ally R, et al. Hepatitis B in subSaharan Africa: strategies to achieve the 2030 elimination targets. Lancet Gastroenterol Hepatol 2017;2:900-9.

DOI: 10.1056/NEJMe1801662

Copyright @ 2018 Massachusetts Medical Society. 